\title{
Role of periostin in esophageal, gastric and colon cancer (Review)
}

\author{
TADEUSZ MONIUSZKO ${ }^{1}$, ANDRZEJ WINCEWICZ ${ }^{2}$, MARIUSZ KODA ${ }^{3}$, \\ IZABELA DOMYSŁAWSKA ${ }^{4}$ and STANISŁAW SULKOWSKI ${ }^{3}$
}

\author{
${ }^{1}$ Department of Respiratory Diagnostics and Bronchofiberoscopy, Medical University of Białystok, Białystok, \\ 15-269 Podlaskie; ${ }^{2}$ Department of Anatomy, Faculty of Health Sciences, Jan Kochanowski University, Kielce, \\ 25-317 Świętokrzyskie; Departments of ${ }^{3}$ General Pathomorphology and ${ }^{4}$ Rheumatology and Internal Diseases, \\ Medical University of Białystok, Białystok, 15-269 Podlaskie, Poland
}

Received August 16,2015; Accepted May 16, 2016

DOI: $10.3892 / 01.2016 .4692$

\begin{abstract}
Periostin, also known as osteoblast-specific factor 2, is a cell-adhesion protein with pleiotropic properties. The protein serves a vital role in the maintenance and development of tooth and bone tissue, in addition to cardiac development and healing. Periostin levels are increased in several forms of cancer, including pancreatic, ovarian, colon, lung, breast, gastric, thyroid, and esophageal head and neck carcinomas. The present review highlights the key role of periostin in tumorigenesis, particularly in increasing cell survival, invasion, angiogenesis, epithelial-mesenchymal transition and metastasis of carcinoma cells by interacting with numerous cell-surface receptors, including integrins, in the phosphoinositide 3-kinase-Akt pathway. In addition, periostin actively affects the canonical Wnt signaling pathway of colorectal tumorigenesis. The current review focused on the involvement of periostin in the development of colorectal, esophageal and gastric cancer.
\end{abstract}

\section{Contents}

1. Introduction

2. Periostin in esophageal cancer

3. Periostin in gastric cancer

4. Periostin in colorectal cancer

5. Conclusion

\section{Introduction}

Periostin, also known as osteoblast-specific factor 2, is an 811 amino acid long protein that is secreted by osteoblasts (1).

Correspondence to: Professor Stanisław Sulkowski, Department of General Pathomorphology, Medical University of Białystok, 13 Waszyngtona Street, Białystok, 15-269 Podlaskie, Poland E-mail: sulek@zeus.net.pl

Key words: periostin, signaling pathways, cancer of alimentary tract, therapy
The protein is a member of the transforming growth factor (TGF)- $\beta$-inducible protein superfamily and is induced in mesenchymal cells following stimulation by TGF- $\beta$, bone morphogenetic protein 2, interleukin (IL)-4 or IL-13 (2-4). Periostin serves a vital role in the maintenance and development of tooth and bone tissue, in addition to cardiac development and healing (2-4).

Potential factors stimulating the secretion of periostin may also include platelet-derived growth factor-AA and -BB, fibroblast growth factor (FGF)-A, -B and -1, and angiotensin II (Ang II). Furthermore, FGF-1 and Ang II enhance periostin expression following activation of the Ras/MEK1/2/extracellular signal-regulated kinase (ERK)1/2, phosphatidylinositol 3-kinase (PI3K)/Akt/p70S6K and Ras/p38 mitogen-activated protein kinase signaling pathways $(5,6)$. Periostin has become a key protein investigated in cancer studies due to its vital function in regulating cell matrix interactions and organization by binding to proteins such as collagen $\mathrm{I} / \mathrm{V}$, fibronectin and tenascin $\mathrm{C}$ (7). The protein also functions as a ligand for integrins, including $\alpha v \beta 3, \alpha v \beta 5$ and $\alpha 6 \beta 4$ (8). Furthermore, periostin interacts with various molecules to affect a number of pathways, such as Notch 1 signaling $(9,10)$, and thus, a lack of periostin may be responsible for a suppression of Notch 1 signaling $(9,10)$. Conversely, it cannot be excluded that the overexpression of periostin may result in the activation of Notch 1 signaling in cancer (10). Additionally, periostin was identified as being involved in gastric cancer cell epithelial-mesenchymal transition (EMT) (11). This study reviews the current state-of-the-art role of periostin in gastrointestinal cancers.

\section{Periostin in esophageal cancer}

A previous study observed that periostin was present in the normal esophagus, but its levels were significantly lower in healthy esophagus tissues than in other areas of the gastrointestinal tract, including the stomach and colorectum (12). Meanwhile, periostin expression was identified to be significantly higher in esophageal squamous cell carcinoma (ESCC) compared with adjacent non-neoplastic esophageal epithelium (13). mRNA profiling of ESCC tissues demonstrated an upregulation of periostin as high as 11-fold (13). Similarly, a quantitative tissue proteomic study reported a 7-fold 
upregulation of periostin in tumor tissues compared with normal tissues (14). However, a further study investigated whether periostin was secreted by cancer cells or non-epithelial cells, including cancer-associated fibroblasts, and it was observed that it was primarily produced by the latter. At the same time, periostin was reported to bind as a ligand to $\alpha v \beta 3$ and $\alpha v \beta 5$ integrins (Fig. 1), thus signaling via the PI3K-Akt pathway within oesophageal adenocarcinoma cells; this indicated that periostin accumulated to a higher degree in ESCC tissue than in adjacent tissue outside the tumor (15).

Luo et al (16) reported that the overexpression of periostin correlated with adventitia invasion, lymph node metastasis and tumor-node-metastasis (TNM) stage. In addition, it was also observed that lymph node metastasis, adventitia invasion and TNM stage were associated with positive expression of synuclein $\gamma$ (breast cancer-specific protein 1) (16), which may be responsible for altering protease secretion and subsequently affecting the tumor biology.

The majority of studies investigating ESCC have focused on the association between periostin and vascular endothelial growth factor (VEGF) and microvessel density (MVD) in the development of cancer and prediction of patient prognosis (17). Periostin-positive tumors reportedly exhibit higher levels of VEGF and MVD compared with periostin-negative tumors (17), with a positive correlation identified between VEGF and periostin; these results indicate that periostin serves a key role in ESCC tumorigenesis through the induction and/or promotion of angiogenesis (17). As high levels of VEGF in serum is associated with a more advanced stage and poorer response to chemoradiotherapy, VEGF may be viewed as a predictive and prognostic factor of further tumor progression and shorter patient survival (18).

Paramount genetic alterations associated with the initiation and progression of ESCC include mutations underlying the overexpression of p53 and epidermal growth factor receptor (EGFR) (19). It was previously demonstrated that culturing EPC2-hTERT-EGFR-p53 ${ }^{\text {R175H }}$ cells, which overexpress EGFR and contain a p53 missense mutation, in a three-dimensional organotypic culture induced the invasion of these cells into the extracellular matrix (20). Michaylira et al (21) reported that the induction of periostin, which was preferentially expressed in invading cells, was dependent upon EGFR signaling and p53 mutation. The restoration of wild-type p53 function was identified as a contributing factor to the attenuation of periostin function in an organotypic culture model of engineered ESCC (22). Esophageal cells overexpressing periostin and mutant $\mathrm{p} 53^{\mathrm{R} 175 \mathrm{H}}$ exhibited an upregulation in the signal transducer and activator of transcription 1 (STAT1) network and STAT-1 dependent genes (22). The knockdown of STAT1 in invasive EPC-hTERT-p53 ${ }^{\mathrm{R} 175 \mathrm{H}}-\mathrm{POSTN}$ and EPC-hTERT-EFGR-P53 ${ }^{\mathrm{R} 175 \mathrm{H}}$ cells resulted in reduced invasiveness, while periostin-knockdown in ESCC tumor xenografts led to a decrease in p53 expression, STAT1 activation and a significant decrease in cluster of differentiation (CD) $44^{\text {hi }} \mathrm{CD} 24^{\text {low }}$ tumor-initiating cells (22).

As periostin levels vary significantly between healthy and diseased tissues, the protein may be used as a distinguishing marker in the diagnosis and therapy of cancer to a certain extent as confirmed by Kwon et al (23) in ESCC. The study focused on the simultaneous involvement of aurora kinase B
(AURKB) and periostin and heat shock protein 47 (HSP47), which each exhibited a significant increase at the mRNA and protein level (23). Notably, AURKB and HSP47 levels were increased during early stages of cancer, whereas periostin levels increased during cell-cell interactions between cancer and adjacent stromal cells (23).

\section{Periostin in gastric cancer}

Normal and benign gastric tissues express periostin at the highest level among the non-neoplastic tissues of other organs of the gastrointestinal tract, with eventual impact on normal epithelium physiology (12). The effect of periostin is considerably more marked in gastric cancer, and is reportedly overexpressed at the mRNA and protein levels in comparison to adjacent notmal tissues (24). Kikuchi et al (25) identified that periostin expression was increased in stage II-IV stomach cancer tissues. By contrast to benign gastric tissues, with cancer progression, the linear deposition of periostin disappeared in intestinal type gastric tubular adenocarcinoma. Furthermore, in situ hybridization documented the presence of periostin expression in fibroblasts, but not in cancer cells (25). Periostin promoted in vitro growth of OCUM-2MLN and OCUM-12 cancer cell lines; this process was associated with ERK activation (25). Finally, the study demonstrated that in vivo mouse fibroblasts with positive periostin expression were susceptible to tumor formation (25).

The varying deposition patterns of periostin in cancer tissue bears testimony to its diverse biological role depending on the source of its origin (26). A recent study by Lv et al (26) demonstrated that the restoration of periostin in gastric cancer cells inhibited their growth and invasiveness. Furthermore, epithelial cell-derived periostin had a positive effect on retinoblastoma protein $(\mathrm{Rb})$ phosphorylation, which induced the activation of the E2F1 target gene $\mathrm{p} 14^{\mathrm{ARF}}(26)$. This gene was responsible for mouse double minute 2 homolog (Mdm2) inactivation, which led to the stabilization of p53 and E-cadherin proteins (26). Notably, under these circumstances, Rb deletion resulted in the abolishment of the aforementioned effects (26).

A further key role of periostin in gastric cancer is the induction of angiogenesis (27). MKN-45 cells under hypoxic conditions exhibited increased periostin expression, which subsequently activated ERK1/2 and resulted in VEGF-mediated angiogenesis (27). When periostin was knocked down in these cells by specific small interfering RNA, this effect was abolished (27).

Nicotine has been identified as an important factor responsible for the induction of gastric cancer, with periostin reported to increase cell invasion, proliferation and EMT in nicotine-induced gastric cancer (11). Nicotine has also been demonstrated to upregulate the expression of periostin in gastric cancer cells via a cyclooxygenase-2-dependent pathway, and thus promotes the mitosis and proliferation of gastric cells (11).

A key issue in chemotherapy is the sensitivity of the cancer cells to the applied drugs (28). SGC-7901 human gastric cancer cells proved susceptible to the apoptosis caused by cisplatin, but overexpression of periostin made these cells more resistant to cisplatin treatment. The same cells treated with 5-fluorouracil (5-FU) behaved in a similar manner (28). Overexpression of 


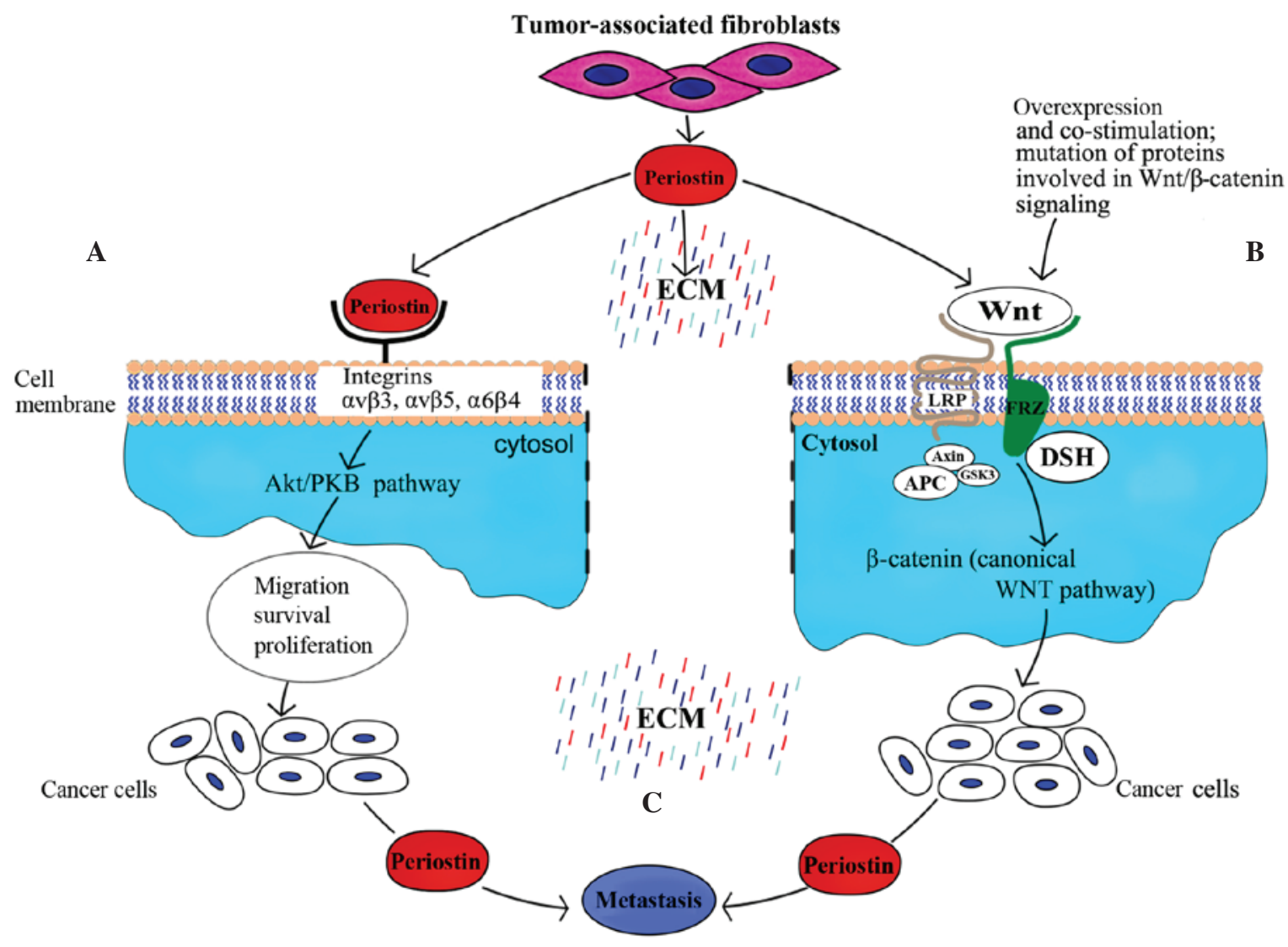

Figure 1. Impact of periostin on cancer with regard to metastatic dissemination. (A) Interaction of periostin with integrins promotes migration, survival and proliferation of cells through activation of the phosphatidylinositol 3-kinase-Akt pathway, which leads to malignant transformation. (B) Periostin activates the canonical Wnt pathway; augmentation of this signal may additionally depend on mutation-related overexpression and co-stimulation with certain oncogenes. (C) Presence of periostin favors the transfer of cancer cells to sites of metastases. PKB, protein kinase B; LRP, low density lipoprotein receptor; DSH, Dishevlled; FRZ, frizzy; APC, adenomatous polyposis coli; GSK3, glycogen synthase kinase 3; ECM, extracellular matrix.

periostin in the SGC-7901 gastric cancer cell line increased resistance to 5-FU or cisplatin-induced apoptosis and reduced the release of cytochrome $c$ from the mitochondria, resulting in diminished cleavage of poly(ADP-ribose) polymerase- 1 and caspase- 3 , and decreased expression of p53 and Bax, in addition to increased Akt phosphorylation and B-cell lymphoma 2 expression (28). It is important to note that the interaction between the p53 and PI3K/Akt pathways is crucial for apoptosis and survival of cancer cells. Furthermore, activation of Akt may result in destabilization of p53 by Mdm2 (29).

\section{Periostin in colorectal cancer}

A relatively high level of periostin was present in normal gastric and colon tissues in comparison to other regions of the alimentary tract, which bears testimony to the role that it plays in the normal physiology of the epithelium (12). Preoperative serum concentrations of periostin were observed to be significantly higher in colorectal cancer (CRC) compared with benign colorectal polyps or adenoma and healthy controls (30). This, in turn, functioned as a marker associated with the oncoming metastasis and generally poor prognosis for the patients (30). Additionally, more advanced stages of the disease were characterized by greater concentrations of periostin (30). CRC tissues exhibited upregulated periostin expression compared with normal tissues, while no periostin mRNA expression was observed in 4 CRC cell lines, including SW480, HT-29, LS174T and SW620 (30). Generally, lower postoperative concentrations of periostin suggest that the increased levels were produced by tumors. Ben et al (30) suggested that periostin may be produced by the stromal cells surrounding the tumor, but not by the CRC cells themselves.

In the normal colon, the highest level of periostin expression is observed in the cells lining the colonic crypts, whilst in $\mathrm{CRC}$, periostin accumulates diffusely in the malignant epithelium. In healthy tissues, mouse and human colonic depositions of periostin are in close proximity to pericryptal fibroblasts, and are also observed in numerous neoplastic tissues (31). Kikuchi et al (31) identified the connection between periostin expression and colorectal crypt fibroblasts and infiltrating fibroblasts of the tumor tissue. Periostin-mediated induction of fibroblasts resulted in an increase in the number of HCT 116 colon cancer cells in co-culture (31).

Regarding studies on periostin, a particularly important observation was the expression and function of the protein in $\mathrm{CD} 133^{+}$tumor cells (32). CD133 is a $120-\mathrm{kD}$ transmembrane glycoprotein, which has been recently employed as a cancer stem cell marker in various types of carcinoma (32). Wu et al (32) observed that $\mathrm{CD} 133^{+}$CRC tumor cells, which possess potent tumorigenicity responsible for tumor initiation and maintenance, were characterized by significantly higher periostin expression compared with CD133- CRC tumor cells. 
Statistical analysis demonstrated a linear correlation between the expression of periostin and clinicopathological factors, including lymph node metastasis, tumor size, histological type, postoperative liver metastasis and TNM stage (32).

Periostin enhances cancer cell survival and promotes their metastatic growth via the Akt/protein kinase B (PKB) pathway (33). As a result, suppression of the Akt/PKB pathway promotes apoptosis through Bad and caspase-9 activation (33). In addition, CRC cell exposure to anti-periostin antibodies results in the activation of apoptosis and potentiation of the effects of 5-FU chemotherapy (33).

Xiao et al (34) reported that HT-29 and SW480 CRC cells treated with 5-FU or oxaliplatin demonstrated greater levels of periostin at the protein and mRNA levels. Notably, periostin was responsible for inhibiting cell apoptosis, and periostin silencing subsequently led to increased apoptosis and enhanced cleavage of caspase- 3 and poly(ADP-ribose) polymerase-1 in the CRC cells following drug treatment (34). Periostin silencing also resulted in the suppressed expression of survivin (34). However, survivin, an antiapoptotic protein present in CRC cells, repressed apoptosis when expressed at low levels in SW480 and HT-29 cells with depleted periostin levels (34). Additionally, overexpression of periostin increased survivin expression and phosphorylation of Akt, which was able to be reversed by pretreatment with the specific inhibitor, LY294002 (34). Periostin interacts with numerous factors at the onset of colon carcinoma, for example, periostin contributes to the increase of Wnt signaling in cancer stem cells by recruiting Wnt ligands, as shown in the experiments by Malanchi et al (35). Furthermore, it was demonstrated that periostin is required for cancer stem cell maintenance and metastasis (35), and participates in the recruitment of Wnt ligands, subsequently increasing Wnt signaling in cancer stem cells (35).

\section{Conclusion}

Periostin promotes cancer growth, not only in primary carcinomas of the esophagus, stomach and large bowel $(11,17,35)$, but also in cancer of other organs, including prostate cancer (36). As neutralization of periostin signaling induces cell apoptosis and enhances the cytotoxic effects of anti-tumor agents in CRC, this protein has emerged as a marker of poor prognosis and a target for anticancer therapy. Construction of novel periostin signaling pathway-targeted drugs could affect the aforemmentioned multiple molecular messengers. These molecular targets should be selected with such modifications that this type of treatment would not disturb the correct functions of healthy cells. However, it is a great challenge to focus the therapeutic property of the targeted drug to exclusively limit cancer growth in the pharmacological regulation of periostin expression, as periostin is a factor that is normally expressed in benign cells and is involved in multiple interactions with numerous other molecules.

\section{References}

1. Takeshita S, Kikuno R, Tezuka $\mathrm{K}$ and Amann E: Osteoblast-specific factor 2: Cloning of a putative bone adhesion protein with homology with the insect protein fasciclin I. Biochem J 294: 271-278, 1993.
2. Horiuchi K, Amizuka N, Takeshita S, Takamatsu H, Katsuura M, Ozawa H, Toyama Y, Bonewald LF and Kudo A: Identification and characterization of a novel protein, periostin, with restricted expression to periosteum and periodontal ligament and increased expression by transforming growth factor beta. J Bone Miner Res 14: 1239-1249, 1999.

3. Takayama G, Arima K, Kanaji T, Toda S, Tanaka H, Shoji S, McKenzie AN, Nagai H, Hotokebuchi T and Izuhara K: Periostin: A novel component of subepithelial fibrosis of bronchial asthma downstream of IL-4 and IL-13 signals. J Allergy Clin Immunol 118: 98-104, 2006.

4. Ji X, Chen D, Xu C, Harris SE, Mundy GR and Yoneda T: Patterns of gene expression associated with BMP-2-induced osteoblast and adipocyte differentiation of mesenchymal progenitor cell 3T3-F442A. J Bone Miner Metab 18: 132-139, 2000.

5. Erkan M,Kleeff J, Gorbachevski A, Reiser C,Mitkus T, Esposito I, Giese T, Büchler MW, Giese NA and Friess H: Periostin creates a tumor-supportive microenvironment in the pancreas by sustaining fibrogenic stellate cell activity. Gastroenterology 132: 1447-1464, 2007

6. Li P, Oparil S, Feng W and Chen YF: Hypoxia-responsive growth factors upregulate periostin and osteopontin expression via distinct signaling pathways in rat pulmonary arterial smooth muscle cells. J Appl Physiol (1985) 97: 1549-1558, 2004.

7. Norris RA, Damon B, Mironov V, Kasyanov V, Ramamurthi A, Moreno-Rodriguez R, Trusk T, Potts JD, Goodwin RL, Davis $\mathrm{J}$, et al: Periostin regulates collagen fibrillogenesis and the biomechanical properties of connective tissues. J Cell Biochem 101: 695-711, 2007.

8. Gillan L, Matei D, Fishman DA, Gerbin CS, Karlan BY and Chang DD: Periostin secreted by epithelial ovarian carcinoma is a ligand for alpha(V)beta(3) and alpha(V)beta(5) integrins and promotes cell motility. Cancer Res 62: 5358-5364, 2002.

9. Tkatchenko TV, Moreno-Rodriguez RA, Conway SJ, Molkentin JD, Markwald RR and Tkatchenko AV: Lack of periostin leads to suppression of Notch1 signaling and calcific aortic valve disease. Physiol Genomics 39: 160-168, 2009.

10. Sriram R, Lo V, Pryce B, Antonova L, Mears AJ, Daneshmand M, McKay B, Conway SJ, Muller WJ and Sabourin LA: Loss of periostin/OSF-2 in ErbB2/Neu-driven tumors results in androgen receptor-positive molecular apocrine-like tumors with reduced Notch1 activity. Breast Cancer Res 17: 7, 2015.

11. Liu Y and Liu BA: Enhanced proliferation, invasion, and epithelial-mesenchymal transition of nicotine-promoted gastric cancer by periostin. World J Gastroenterol 17: 2674-2680, 2011.

12. Tai IT, Dai M and Chen LB: Periostin induction in tumor cell line explants and inhibition of in vitro cell growth by anti-periostin antibodies. Carcinogenesis 26: 908-915, 2005.

13. Pawar H, Kashyap MK, Sahasrabuddhe NA, Renuse S, Harsha HC, Kumar P, Sharma J, Kandasamy K, Marimuthu A, Nair B, et al: Quantitative tissue proteomics of esophageal squamous cell carcinoma for novel biomarker discovery. Cancer Biol Ther 12: 510-522, 2011.

14. Kashyap MK, Marimuthu A, Kishore CJ, Peri S, Keerthikumar S, Prasad TS, Mahmood R, Rao S, Ranganathan P, Sanjeeviah RC, et al: Genomewide mRNA profiling of esophageal squamous cell carcinoma for identification of cancer biomarkers. Cancer Biol Ther 8: 36-46, 2009.

15. Underwood TJ, Hayden AL, Derouet M, Garcia E, Noble F, White MJ, Thirdborough S, Mead A, Clemons N, Mellone M, et al: Cancer-associated fibroblasts predict poor outcome and promote periostin-dependent invasion in oesophageal adenocarcinoma. J Pathol 235: 466-477, 2015.

16. Luo JH, Zhou J and Gao Y: Correlation between periostin and SNCG and esophageal cancer invasion, infiltration and apoptosis. Asian Pac J Trop Med 6: 516-519, 2013.

17. Wang W, Sun QK, He YF, Ma DC, Xie MR, Ji CS and Hu B: Overexpression of periostin is significantly correlated to the tumor angiogenesis and poor prognosis in patients with esophageal squamous cell carcinoma. Int J Clin Exp Pathol 7: 593-601, 2014.

18. Cheng JC, Graber MS, Hsu FM, Tsai CL, Castaneda L, Lee JM, Chang DT and Koong AC: High serum levels of vascular endothelial growth factor-A and transforming growth factor- $\beta 1$ before neoadjuvant chemoradiotherapy predict poor outcomes in patients with esophageal squamous cell carcinoma receiving combined modality therapy. Ann Surg Oncol 21: 2361-2368, 2014.

19. Shang L, Liu HJ, Hao JJ, Jiang YY, Shi F, Zhang Y, Cai Y, Xu X, Jia XM, Zhan QM and Wang MR: A panel of overexpressed proteins for prognosis in esophageal squamous cell carcinoma. PLoS One 9: e111045, 2014. 
20. Okawa T, Michaylira CZ, Kalabis J, Stairs DB, Nakagawa H, Andl CD, Johnstone CN, Klein-Szanto AJ, El-Deiry WS, Cukierman E, et al: The functional interplay between EGFR overexpression, hTERT activation, and p53 mutation in esophageal epithelial cells with activation of stromal fibroblasts induces tumor development, invasion, and differentiation. Genes Dev 21: 2788-2803, 2007.

21. Michaylira CZ, Wong GS, Miller CG, Gutierrez CM, Nakagawa H, Hammond R, Klein-Szanto AJ, Lee JS, Kim SB, Herlyn M, et al: Periostin, a cell adhesion molecule, facilitates invasion in the tumor microenvironment and annotates a novel tumor-invasive signature in esophageal cancer. Cancer Res 70: 5281-5292, 2010.

22. Wong GS, Lee JS, Park YY, Klein-Szanto AJ, Waldron TJ, Cukierman E, Herlyn M, Gimotty P, Nakagawa H and Rustgi AK: Periostin cooperates with mutant p53 to mediate invasion through the induction of STAT1 signaling in the esophageal tumor microenvironment. Oncogenesis 2: e59, 2013.

23. Kwon YJ, Lee SJ, Koh JS, Kim SH, Kim YJ and Park JH: Expression patterns of aurora kinase B, heat shock protein 47 and periostin in esophageal squamous cell carcinoma. Oncol Res 18: 141-151, 2009.

24. Li JS, Sun GW, Wei XY and Tang WH: Expression of periostin and its clinicopathological relevance in gastric cancer. World J Gastroenterol 13: 5261-5266, 2007.

25. Kikuchi Y, Kunita A, Iwata C, Komura D, Nishiyama T, Shimazu K, Takeshita K, Shibahara J, Kii I, Morishita Y, et al: The niche component periostin is produced by cancer-associated fibroblasts, supporting growth of gastric cancer through ERK activation. Am J Pathol 184: 859-870, 2014.

26. Lv H, Liu R, Fu J, Yang Q, Shi J, Chen P, Ji M, Shi B and Hou P. Epithelial cell-derived periostin functions as a tumor suppressor in gastric cancer through stabilizing p53 and E-cadherin proteins via the $\mathrm{Rb} / \mathrm{E} 2 \mathrm{~F} 1 / \mathrm{p} 14 \mathrm{ARF} / \mathrm{Mdm} 2$ signaling pathway. Cell Cycle 13: 2962-2974, 2014.
27. Qiu F, Shi CH, Zheng J and Liu YB: Periostin mediates the increased pro-angiogenic activity of gastric cancer cells under hypoxic conditions. J Biochem Mol Toxicol 27: 364-369, 2013.

28. Li B, Wang L and Chi B: Upregulation of periostin prevents P53-mediated apoptosis in SGC-7901 gastric cancer cells. Mol Biol Rep 40: 1677-1683, 2013.

29. Haupt S, Berger M, Goldberg Z and Haupt Y: Apoptosis - the p53 network. J Cell Sci 116: 4077-4085, 2003.

30. Ben QW, Zhao Z, Ge SF, Zhou J, Yuan F and Yuan YZ: Circulating levels of periostin may help identify patients with more aggressive colorectal cancer. Int J Oncol 34: 821-828, 2009.

31. Kikuchi Y, Kashima TG, Nishiyama T, Shimazu K, Morishita Y, Shimazaki M, Kii I, Horie H, Nagai H, Kudo A and Fukayama M: Periostin is expressed in pericryptal fibroblasts and cancer-associated fibroblasts in the colon. J Histochem Cytochem 56 753-764, 2008

32. Wu G, Wang X and Zhang X: Clinical implications of periostin in the liver metastasis of colorectal cancer. Cancer Biother Radiopharm 28: 298-302, 2013

33. Bao S, Ouyang G, Bai X, Huang Z, Ma C, Liu M, Shao R, Anderson RM, Rich JN and Wang XF: Periostin potently promotes metastatic growth of colon cancer by augmenting cell survival via the Akt/PKB pathway. Cancer Cell 5: 329-339, 2004.

34. Xiao ZM, Wang XY and Wang AM: Periostin induces chemoresistance in colon cancer cells through activation of the PI3K/Akt/survivin pathway. Biotechnol Appl Biochem 62: 401-406, 2015.

35. Malanchi I, Santamaria-Martínez A, Susanto E, Peng H, Lehr HA, Delaloye JF and Huelsken J: Interactions between cancer stem cells and their niche govern metastatic colonization. Nature 481: 85-89, 2011.

36. Argellati F, Nuzzo PV, Ricci F, Mangerini R, Rubagotti A and Boccardo F: Dihydrotestosterone and bicalutamide do not affect periostin expression in androgen-dependent $\mathrm{LNCaP}$ prostate cancer cell lines. Anticancer Res 33: 815-820, 2013. 